\title{
Tuberkulose im Gesundheitswesen
}

\author{
Tuberculosis in Healthcare Workers
}

Autor

Institut

\section{A. Nienhaus}

Berufsgenossenschaft für Gesundheitsdienst und Wohlfahrtspflege, Fachbereich Gesundheitsschutz, Hamburg
Bibliografie

DOI $10.1055 / \mathrm{s}-0028-1100828$

Online-Publikation: 9.1.2009

Pneumologie 2009; 63: 23-30

(c) Georg Thieme Verlag KG

Stuttgart · New York

ISSN 0934-8387

Korrespondenzadresse

Priv.-Doz. Dr. med.

Albert Nienhaus

Berufsgenossenschaft für

Gesundheitsdienst und

Wohlfahrtspflege,

Fachbereich Gesundheitsschutz

Pappelallee 35/37

22089 Hamburg

albert.nienhaus@bgw-online.de

Serienherausgeber

R. Loddenkemper, Berlin

T. Schaberg, Rotenburg

\section{Zusammenfassung \\ $\nabla$}

Die Wahrnehmung des beruflichen TuberkuloseInfektionsrisikos für Beschäftigte im Gesundheitswesen hat sich in den letzten Jahren tiefgreifend verändert. Insbesondere molekular-epidemiologische Studien mittels Fingerprinting, aber auch eine systematische Auswertung konventioneller Studien zum Infektionsrisiko legen nahe, dass viele der Tuberkulose (Tbc)-Erkrankungen bei Beschäftigten im Gesundheitswesen beruflich bedingt sind. In der Hamburger Fingerprintstudie waren $80 \%$ der Tbc-Erkrankungen bei Beschäftigten im Gesundheitsdienst durch eine beruflich erworbene Übertragung verursacht. In einer vergleichbaren niederländischen Studie waren es $43 \%$. Neben den typischen Einrichtungen, in denen Tbc-Patienten behandelt werden oder Sputumproben untersucht werden, bestehen erhöhte Infektionsrisiken auch für den Rettungsdienst, die Notaufnahme und die Betreuung von Risikogruppen (Obdachlose, Drogensüchtige, Immigranten aus Gebieten mit hoher Tbc-Inzidenz) sowie bei Beschäftigung in der Altenpflege. Im Berufskrankheitenverfahren gelten diese Beschäftigtengruppen als infektionsgefährdet, auf den Nachweis einer Infektionsquelle (Indexperson) kann deshalb verzichtet werden. Auch wenn der Anteil beruflich verursachter Tuberkulose-Erkrankungen bei Beschäftigten im Gesundheitswesen höher ist als zuvor angenommen, weisen die laufenden Studien zur Evaluation der Interferon- $\gamma$-Release Assays darauf hin, dass die Prävalenz der latenten Tuberkulose-Infektion (LTBI) bei Beschäftigten im Gesundheitsdienst mit etwa $10 \%$ geringer ist als bisher angenommen. Präventionsstrategien zur Vermeidung beruflich bedingter Tuberkulosen müssen daher überdacht werden.

\section{Abstract \\ $\nabla$}

Perception and knowledge of the TB-infection risk in healthcare workers (HCWs) changed profoundly in Germany during the past few years. Molecular-epidemiological studies and a comprehensive review of the existing evidence concerning the infection risk for HCWs lead to the conclusion that TB in HCWs is often caused by infection at the workplace. In the Hamburg Fingerprint Study, $80 \%$ of the TB cases in HCWs were caused by infections at the workplace. In a similar Dutch study $43 \%$ of all cases were work-related. Besides of the well-known risks in TB wards and laboratories, an increased risk for infection should be assumed for paramedics, in emergency rooms, for HCWs caring for the elderly or for workers with close contact to high-risk groups (homeless people, i.v. drug users, migrants from high-incidence countries). TB in a HCW working in these fields can be recognised as an occupational disease (OD) without identifying a particular source of infection. For all other HCWs, the German occupational disease law requires the identification of a source case before TB in an HCW can be accepted as an OD. Even though the proportion of work-related TB in HCWs is higher than was assumed before previously, the prevalence of latent TB infection (LTBI) is lower than expected. In an ongoing evaluation study of the interferon- $\gamma$ release assay (IGRA) LTBI prevalence in HCWs is $10 \%$. Prevention strategies in Germany should be reconsidered in the light of these new findings. 


\section{Einleitung \\ $\nabla$}

Die Zahl der Tuberkulose (Tbc)-Neuerkrankungen ist in Deutschland seit den 1950er-Jahren von rund 130000 jährlich registrierten Tbc-Fällen [1] auf 5402 im Jahr 2006 zurückgegangen. Im Vergleich zu 2005 ist das ein Rückgang um 10\% [2]. Mit dem deutlich selteneren Auftreten der Tbc in der Allgemeinbevölkerung, so ein oft angeführtes Argument, sei auch das Risiko, berufsbedingt an Tbc zu erkranken, vernachlässigbar. „Die Annahme, eine Tätigkeit im Gesundheitswesen als solche bringe eine erhöhte Tbc-Infektionsgefährdung mit sich, hat angesichts des Tuberkulose-Rückgangs keine Berechtigung mehr“" [3]. Ähnlich argumentierten auch Jentgens und Wandelt-Freerksen [4], deren Arbeit für die Begutachtung der Tbc als Berufskrankheit für lange Zeit Richtung gebend war. Belegt durch epidemiologische Studien werden diese Aussagen allerdings nicht.

Die Zunahme der Tbc in den Ländern der ehemaligen Sowjetunion (Neue Unabhängige Staaten der ehemaligen Sowjetunion, NUS) sowie in Subsahara-Afrika, aber auch das vermehrte Auftreten von multiresistenten Tbc-Stämmen, insbesondere in den osteuropäischen Ländern [5], hat infolge der gleichzeitig zunehmenden Migration aus den ökonomisch gebeutelten NUS-Ländern auch in Deutschland die Tbc in den letzten Jahren wieder verstärkt ins öffentliche Bewusstsein gerückt. Belegt ist, dass die multiresistenten Tbc-Stämme insbesondere aus den NUSStaaten eingeführt werden [6].

Das Auftreten der Tbc als Ko-Infektion bei HIV und AIDS hat in den USA, die schon seit längerem zu den Niedrig-Inzidenz-Ländern zählen, die Diskussion um ein berufliches Infektionsrisiko neu belebt und in diesem Zusammenhang zu einer Vielzahl von epidemiologischen Studien geführt [7]. Neue infektionsepidemiologische Erkenntnisse aus so genannten Clusteranalysen mittels Fingerprinting zur Stammdifferenzierung sprechen dafür, dass die Bedeutung der Reaktivierung früher erworbener tuberkulöser Infektionen geringer einzuschätzen ist als bisher angenommen. Der Anteil der Neuinfektionen beträgt in Industrieländern nicht etwa $10 \%[8,9]$, wie bislang angenommen, sondern vermutlich bis zu 40\% [10-12]. Allerdings lässt sich auch zeigen, dass durch gezielte Präventionsmaßnahmen in Risikogruppen der Anteil frischer Übertragungen als Ursache für eine aktive Tbc deutlich gesenkt werden kann [13].

In dieser Arbeit wird das berufliche Infektionsrisiko von Beschäftigten im Gesundheitsdienst neu bewertet. Grundlage hierfür bildet eine Analyse der epidemiologischen Studien zum Infektionsrisiko aus Ländern mit niedriger Tbc-Inzidenz. Insbesondere werden die molekular-epidemiologischen Studien mittels Fingerprinting gewürdigt und Angaben zur Häufigkeit der latenten Tbc-Infektion (LTBI) bei Beschäftigten im Gesundheitswesen, basierend auf Studien zur Evaluation des Interferon- $\gamma$-Release Assays (IGRA), gemacht. Abgerundet wird die Arbeit durch Ausführungen zum Berufskrankheitenverfahren. Dargelegt werden die rechtlichen Grundlagen der Beweiserleichterung und die Kriterien für deren Gewährung.

\section{Konventionelle Untersuchungen zum beruflichen Tbc-Risiko $\nabla$}

In Tab. 1 sind Studien zur beruflichen Tbc-Morbidität und Konversion im Tuberkulin-Hauttest (THT) zusammengefasst. Auf eine Darstellung der einzelnen Studien wird hier aus Platzgründen verzichtet. Eine ausführliche Darstellung der Studien findet sich, abgesehen von 5 neuen Studien [14-18], bei Seidler und Nienhaus [19] sowie bei Seidler u. Mitarb. [20]. Abgeleitet werden sollte eine Aussage zum berufsbezogenen TuberkuloseRisiko in Regionen mit niedriger Tbc-Inzidenz. Daher wurden Studien aus Regionen mit einer Tbc-Inzidenz von über 50/100000 ebenso wie Studien in Krankenhäusern mit mehr als 50 Tuberkulose-Patienten pro 10000 Zugängen bzw. in Krankenhäusern mit 200 oder mehr Tuberkulose-Patienten pro Jahr aus der folgenden Darstellung ausgeschlossen. Nicht berücksichtigt wurden außerdem Studien zu „Outbreaks“, da diese Studien die Möglichkeit einer Übertragung zwar belegen, zum beruflichen Infektionsrisiko aber keine Aussage zulassen.

Die zusammenfassende Bewertung in $\bullet$ Tab. 1 erfolgt getrennt für die einzelnen Berufsgruppen und getrennt nach der methodischen Qualität. Die Anzahl der Studien wird in folgender Reihenfolge aufgelistet: Studien mit guter Qualität, Studien mit akzeptabler Qualität und Studien mit unzureichender Qualität. Unterschieden wird ferner zwischen Studien, die ein signifikantes und deutlich erhöhtes Relatives Risiko ( $R R \geq=2$ ), ein signifikantes, jedoch nicht deutlich erhöhtes Relatives Risiko $(R R<2)$ aufweisen, sowie Studien, die keinen oder einen signifikant negativen Zusammenhang ergeben.

In den 6 Studien mit guter methodischer Qualität wurden für 15 verschiedene Berufsgruppen oder Tätigkeiten das Konversionsbzw. das Erkrankungsrisiko untersucht. In der Mehrzahl der untersuchten Zusammenhänge ergab sich ein erhöhtes Tbc-Risiko (12 von 15 Berufsgruppen). Das Infektionsrisiko von Pflegekräften (ohne weitere Differenzierung) wurde in 4 guten Studien untersucht. In allen 4 Studien [21 - 24] fand sich ein statistisch signifikant erhöhtes Relatives Risiko >2. Dieser Zusammenhang kann deshalb als relativ gesichert betrachtet werden. Das Infektionsrisiko für Ärzte im Allgemeinen wurde in 2 guten Studien untersucht [21,23]. Die Ergebnisse widersprechen sich allerdings. Während eine Studie [23] ein Relatives Risiko größer als 2 fand, beobachtete die andere Studie [21] keinen statistisch signifikanten Zusammenhang. Eine Studie mit akzeptabler Qualität [14] fand ein statistisch signifikant erhöhtes Risiko ( $R R<2), 2$ Studien $[25,26]$ fanden keinen statistisch signifikanten Zusammenhang und eine Studie fand ein statistisch signifikant erniedrigtes Relatives Risiko [27]. Die vorhandenen epidemiologischen Studien verweisen in der Gesamtschau daher nicht auf ein erhöhtes Risiko für Ärzte allgemein. Für Aussagen zu speziellen ärztlichen Fachdisziplinen liegen keine guten epidemiologischen Studien vor. Auch die 8 akzeptablen Studien [14,28-34] sind nicht ausreichend für eine differenzierte Beurteilung. Zwar verweisen 9 von 12 Untersuchungen für spezielle Arztgruppen auf ein erhöhtes Infektionsrisiko, da sich diese 9 Zusammenhangsuntersuchungen aber auf 7 ärztliche Berufsgruppen verteilen, erlauben sie mit einer Ausnahme keine belastbaren Aussagen. Die am besten untersuchte ärztliche Berufsgruppe ist mit 4 methodisch akzeptablen Studien die der Pathologen. Von den 4 Studien fanden 2 ein deutlich erhöhtes Relatives Risiko [30,32] und eine Studie ein um 60\% erhöhtes statistisch signifikantes Risiko [31], wohingegen nur eine Studie keinen statistischen $\mathrm{Zu}-$ sammenhang feststellte [34].

Eine gute [22] und 2 akzeptable Studien [35,36] fanden für Beschäftigte auf Stationen mit Tbc-Patienten ein statistisch signifikantes, deutlich erhöhtes Tbc-Risiko $(R R>2)$. Eine weitere akzeptable Studie fand ein erhöhtes, aber nicht statistisch signifikantes Risiko [37]. Für Beschäftigte auf Stationen, wo bekanntermaßen Tbc-Patienten behandelt werden, ist ein erhöhtes Infektionsrisiko damit epidemiologisch gut gesichert. 
Tab.1 Anzahl der epidemiologischen Studien nach Risikokategorien (in folgender Reihenfolge aufgelistet: Anzahl der Studien zum Tbc-Infektions- und/oder Erkrankungsrisiko nach Risiko-Kategorie (in folgender Reihenfolge aufgelistet): Studien mit guter Qualität/Studien mit akzeptabler Qualität/Studien mit unzureichender Qualität, aktualisiert nach [19]

\begin{tabular}{|c|c|c|c|c|}
\hline & $\begin{array}{l}\text { Signifikant und deutlich } \\
(R R>2) \text { erhöht }\end{array}$ & $\begin{array}{l}\text { Signifikant, } \\
R R<2\end{array}$ & $\begin{array}{l}\text { Kein } \\
\text { Zusammenhang }\end{array}$ & $\begin{array}{l}\text { Signifikant negativer } \\
\text { Zusammenhang }\end{array}$ \\
\hline Medizinische Berufe allgemein & $1 / 1 /-$ & & $-/ 1 / 5$ & $-|-| 1$ \\
\hline $\begin{array}{l}\text { Krankenhausbeschäftigte mit häufigem } \\
\text { Patientenkontakt }\end{array}$ & $-1-13$ & & $-12 / 2$ & $-\mid 1 /-$ \\
\hline $\begin{array}{l}\text { Beschäftigte auf Stationen mit Tuberkulose- } \\
\text { Patienten }\end{array}$ & $1 / 2 / 1$ & $-\mid-/ 1$ & & \\
\hline $\begin{array}{l}\text { Kontakt mit Patienten mit initial } \\
\text { unbekannter Tuberkulose }\end{array}$ & & $(1) * /-1-$ & $(1) * /-\mid 1$ & \\
\hline Pflegekräfte allgemein & $4 / 2 / 1$ & $-|4|-$ & $-/ 3 / 4$ & $-\mid-/ 1$ \\
\hline Pflegekräfte auf Innerer Station & $-\mid 1 /-$ & & & \\
\hline Betreuung HIV-positiver Patienten & $-/ 2 / 1$ & $-|-| 1$ & $-|1|-$ & \\
\hline Betreuung drogenabhängiger Patienten & $-|1|-$ & & & \\
\hline Notaufnahme/ Notfallabteilung & $-|1|-$ & & & \\
\hline Ärzte allgemein & $1 /-/ 1$ & $-\mid 1 /-$ & $1 / 2 / 4$ & $-\mid 1 /-$ \\
\hline Internisten & $-|2|-$ & & $-|-| 1$ & \\
\hline Anästhesisten & $-\mid 1 /-$ & & & \\
\hline Pneumologen & & & $-\mid-/ 1$ & \\
\hline Chirurgen & $-|2|-$ & & $-1-12$ & \\
\hline Psychiater & $-\mid 1 /-$ & & & \\
\hline Pädiater/Pädiatrie-Beschäftigte & & & $-/ 1 / 2$ & \\
\hline Radiologen & & $-\mid 1 /-$ & $-\mid-/ 1$ & $-\mid 1 /-$ \\
\hline Zahnärzte & & & $-\mid-/ 1$ & \\
\hline Pathologen/in der Pathologie Beschäftigte & $-/ 2 / 1$ & $-\mid 1 /-$ & $-/ 1 / 2$ & \\
\hline Laborbeschäftigte & $-/ 1 / 2$ & $-|1|-$ & $-/ 1 / 3$ & \\
\hline $\begin{array}{l}\text { Bestattungsunternehmer/in Bestattungs- } \\
\text { unternehmen Beschäftigte }\end{array}$ & $-|2|-$ & & & \\
\hline Atemtherapeuten & $1 / 3 / 1$ & & $1 /-\mid-$ & \\
\hline Physikalische Therapie & $1 /-/-$ & & & \\
\hline Wäschereibeschäftigte & $-\mid 1 /-$ & & & \\
\hline Küchenpersonal & & $-\mid 1 /-$ & $-\mid-/ 1$ & \\
\hline Hauswirtschaftliche Beschäftigte & $1 / 1 / 1$ & & $-\mid-/ 1$ & \\
\hline Wachpersonal und Transportdienst & $-\mid 1 /-$ & & & \\
\hline $\begin{array}{l}\text { Gefängnisangestellte/Beschäftigte mit } \\
\text { Kontakt zu Gefängnisinsassen }\end{array}$ & $-|2|-$ & & $-/ 1 / 1$ & \\
\hline $\begin{array}{l}\text { Schutzmaßnahmen zur Senkung der } \\
\text { Infektions-/Erkrankungsrate }\end{array}$ & $1 / 1 / 1$ & & $-1-12$ & \\
\hline
\end{tabular}

Zusammenfassend überwiegen bei den methodisch guten oder akzeptablen Studien diejenigen, die ein erhöhtes Tbc-Risiko für verschiedene Tätigkeiten im Gesundheitswesen belegen (53 von 72 Zusammenhangsuntersuchungen). Die bisher für die Begutachtung der Tbc im Berufskrankheitenverfahren gängige Praxis, „eine besondere Ansteckungsgefahr (...) in Praxen und Infektionsabteilungen mit gehäufter Behandlung Tuberkulosekranker oder in bakteriologischen Laboratorien, die sich speziell mit Züchtung und Typisierung von Tuberkelbakterien beschäftigen“, anzunehmen [3] und für alle anderen Bereiche den Nachweis einer Indexperson als Infektionsquelle zu fordern, erscheint aufgrund der Analyse der konventionellen Studien zum beruflichen Infektionsrisiko daher nicht gerechtfertigt.

\section{Molekular-epidemiologische Untersuchungen zum Tbc-Risiko $\nabla$}

Das Fingerprinting und die ergänzenden mikrobiologischen Verfahren zur Stammdifferenzierung des Mycobacterium tuberculosis verbessern die Möglichkeit zur Auffindung von Infektionspfaden [38]. Personen mit unterschiedlichem Tbc-Stamm können sich nicht gegenseitig infiziert haben, während Personen mit gleichem Tbc-Stamm möglicherweise einer gemeinsamen Infektionskette angehören. In der Molekularepidemiologie zur TbcÜbertragung spricht man bei Patienten mit gemeinsamem TbcStamm von „Clustern“. Die Zugehörigkeit zu einem Cluster kann aber nicht mit einer gesicherten Übertragung der Tbc gleichgesetzt werden. Vielmehr muss durch Befragung der Betroffenen ein entsprechender infektiöser Kontakt wahrscheinlich gemacht werden. Bisher wurden 5 molekular-epidemiologische Studien zum beruflichen Infektionsrisiko publiziert [39-43].

Die beiden älteren Studien sind reine Cluster-Analysen ohne weitere Untersuchung der möglichen Infektionspfade bei den Personen eines Cluster $[39,40]$. Die beiden Studien sind daher 
in ihrer Aussagekraft sehr beschränkt. Im Ergebnis widersprechen sie sich. Die amerikanische Fingerprint-Analyse [39] fand ein adjustiertes Odds Ratio von 2,77 (95\% CI: 1,19-6,41) für eine Clusterzugehörigkeit von Tbc-Patienten mit medizinischen Berufen im Vergleich zu solchen mit nicht-medizinischen Berufen. In der bevölkerungsbezogenen, holländischen Studie [40] waren Beschäftigte im Gesundheitswesen signifikant seltener einem Cluster zuzuordnen als andere Beschäftigte (adjustierte Odds Ratio $=0,11 ; 95 \% \mathrm{CI}$ : 0,02-0,71).

In der Hamburger Fingerprintstudie [41] werden seit 1997 fortlaufend von allen meldepflichtigen Tbc-Erkrankungen mit einer Mykobakterien-Kultur Fingerprints erstellt. Zusätzlich zur Erstbefragung im Rahmen der Umgebungsuntersuchung wird bei Mitgliedern eines Clusters, also bei Erkrankten mit gleichem Fingerprintmuster, eine Zweitbefragung zu möglichen Kontakten unter den Clustermitgliedern durchgeführt. Die Studie umfasste am 31.12.2002 insgesamt 848 Fälle, bei denen Fingerprintings mittels IS6110 durchgeführt werden konnten. 10 an Tbc erkrankte Studienteilnehmer waren im Gesundheitsdienst beschäftigt. Von diesen konnte bei 8 Personen über das Fingerprinting und die anschließende Befragung eine frische beruflich bedingte Übertragung nachgewiesen werden. Der Anteil der beruflich verursachten Tuberkulosen betrug bei Beschäftigen im Gesundheitswesen also $80 \%$ (95\%CI 44-97\%). Von den 8 beruflich bedingten Übertragungen waren 5 vor dem Fingerprinting nicht bekannt.

In einer Fingerprintstudie zum Infektionsrisiko bei Beschäftigten im Gesundheitsdienst in den Niederlanden wurde bei 28 von 67 (43\%) aktiven Tuberkulosen eine Übertragung von Patienten auf das Personal nachgewiesen [42]. Die Hauptursache für die Übertragung war die verspätete Diagnosestellung der Indexfälle besonders bei älteren Patienten. $44 \%$ der Indexfälle waren älter als 60 Jahre. 10 (von 28) Indexfälle waren ältere Menschen mit vorhandener Komorbidität, bei denen die Tuberkulose nicht zeitnah diagnostiziert wurde und deshalb keine adäquaten Präventionsmaßnahmen getroffen wurden.

In der aktuellsten molekular-epidemiologischen Studie zum beruflichen Infektionsrisiko wurden alle in San Francisco gemeldeten Tbc-Fälle aus den Jahren von 1993-2003 einbezogen [43]. Insgesamt gab es 2510 Tbc-Fälle. Ein anschließendes Fingerprint konnte bei 1852 Patienten erstellt werden. 31 Patienten (1,2\% aller Tbc-Fälle) arbeiteten im Gesundheitswesen. Eine beruflich verursachte Tbc-Erkrankung wurde bei 10 (32\%) der 31 HCW bestätigt. Bei 4 von 8 Beschäftigten, die zu einem Cluster gehören, war die Tuberkulose beruflich verursacht. In 4 von 16 Fällen ohne Cluster sowie bei 2 Patienten ohne Fingerprinting war die Tbc-Infektion ebenfalls beruflich verursacht.

Es lassen sich keine weiteren bevölkerungsbezogenen molekularbiologischen Studien identifizieren, die den Anteil der ClusterZugehörigkeit getrennt für einzelne Berufsgruppen angeben. Mehrere Fallbeschreibungen und „Ausbruchs-Analysen“ weisen mit molekularbiologischen Methoden auf die berufsbezogene Übertragung von Tuberkulose hin. Betroffen sind KrankenhausBeschäftigte [43 -46], Laborbeschäftigte [47], Pathologie-Mitarbeiter [48] und Beschäftigte in der Verwertung medizinischen Abfalls [49].

Zum Teil geben molekular-epidemiologische Studien auch indirekt Aufschluss auf das berufliche Infektionsrisiko. Einer Studie zur molekularen Tuberkulose-Epidemiologie in San Francisco entstammt die Analyse der Infektiosität mikroskopisch sputumnegativer Tuberkulose-Patienten [50]. Mindestens 17\% der Tuberkulose-Fälle konnten in dieser Studie auf eine sputum-nega- tive Infektionsquelle zurückgeführt werden. Zwar würden sputum-negative Patienten weniger Bakterien verbreiten, jedoch trägt die verzögerte Diagnose (in der beschriebenen Studie durchschnittlich 10 Tage später) und die fehlende Isolierung von sputum-negativen Patienten zur Infektiosität bei. Auch in der Untersuchung des „Tuberculosis Genotyping Network“ waren $19 \%$ der mit konventionellen Methoden der Kontaktevaluation vermuteten und molekularbiologisch bestätigten Übertragungszusammenhänge auf sputum-negative Infektionsquellen zurückzuführen [51]. Die Möglichkeit der Übertragung durch sputum-negative Patienten wird auch durch eine englische Studie [52] bestätigt.

\section{Prävalenz der latenten Tbc-Infektion (LTBI) bei Beschäftigten im Gesundheitswesen}

$\nabla$

Mit den Interferon- $\gamma$-Release Assays (IGRA) stehen nun Methoden zur Verfügung, die eine deutlich bessere Spezifität aufweisen als der Tuberkulin-Hauttest (THT) nach Mendel-Mantoux [53]. Die Gefahr der Überschätzung der LTBI-Prävalenz aufgrund von Kreuzreaktionen mit der BCG-Impfung, dem Kontakt zu Umweltmykobakterien oder anderer unspezifischer Reaktionen besteht bei diesen Tests nicht [54]. In einer Evaluationsstudie bei Beschäftigten im Gesundheitswesen waren $24 \%$ positiv im THT nach Mendel-Mantoux ( $>5 \mathrm{~mm}$ ), aber nur $10 \%$ positiv im IGRA [55]. 47 von 48 Beschäftigten mit einem positiven THT aber negativen IGRA waren BCG geimpft oder hatten einen vorherigen THT. Eine dänischen Studie berichtet ebenfalls eine sehr niedrige Prävalenz an LTBI bei Beschäftigten im Gesundheitswesen: 2 von 139 (1\%). Im THT nach Mendel-Mantoux waren hingegen $34 \%$ positiv [56].

- Tab. 2 gibt die neueren Zwischenergebnisse der oben genannten fortlaufenden deutschen Studie [54] wieder. Die Prävalenz der LTBI bei Beschäftigten aus 6 verschiedenen Krankenhäusern beträgt $10 \%$, wenn sie mit dem IGRA bestimmt wird [57]. Sie ist stark abhängig vom Alter. Bei jungen Beschäftigten (<30 Jahre) ist die Prävalenz der LTBI niedrig (3\%). Von den über 60-Jährigen war jeder zweite infiziert (56,5\%). Diese Unterschiede sind wahrscheinlich durch einen Kohorten-Effekt zu erklären. D.h. Personen, die früher geboren wurden (nach dem 2. Weltkrieg), haben ein höheres Risiko infiziert zu sein, als Personen, die später in den Jahren einer deutlich niedrigeren TB-Inzidenz geboren sind. Immigranten hatten eine höhere Prävalenz als in Deutschland geborene Beschäftigte (OR 2,4; $95 \%$ CI 1,4-4,2). Ärzte, Krankenschwestern und nicht medizinisches Personal hatten vergleichbare Prävalenzraten. 53\% derjenigen mit einem positiven IGRA hatten einen negativen THT $(0-4 \mathrm{~mm})$. Sie wären also bei einer Strategie, die den IGRA nur bei positivem THT einsetzt, nicht entdeckt worden.

In der Literatur wird diskutiert, dass die höhere Rate an positiven Ergebnissen im THT daran liegen könnte, dass der THT auch auf alte Infektionen reagiert, während der IGRA eher neue Infektionen findet. Diese Hypothese passt aber nicht zu der Beobachtung, dass Probanden mit negativem THT und positivem IGRA eher älter waren, als Patienten mit positivem THT, aber negativem IGRA [55]. Die Analyse von Risikofaktoren für diskordante Ergebnisse zwischen dem THT und dem IGRA ergab, dass sich in Deutschland THT-negative/IGRA-positive-Kombinationen zu 54\% durch ein Alter größer als 40 Jahre der Testpersonen und THT-positiv/IGRA-negativ-Ergebnisse zu 95\% durch BCG-Impfungen und/oder Migrationshintergrund erklären lassen [58]. 
Tab.2 Adjustierte Odds Ratios für positiven IGRA ( $n=821)$

\begin{tabular}{|c|c|c|c|c|}
\hline Alter & $\begin{array}{l}\text { IGRA } \\
\text { negativ } \\
\text { n (\%) }\end{array}$ & $\begin{array}{l}\text { positiv } \\
\text { n (\%) }\end{array}$ & Odds Ratio* & $95 \% \mathrm{Cl}$ \\
\hline$<30$ Jahre & $230(97,0)$ & $7(3,0)$ & 1 & - \\
\hline 30-39 Jahre & $218(94,8)$ & $12(5,2)$ & 1,4 & $0,5-3,7$ \\
\hline 40-49 Jahre & $196(86,3)$ & $31(13,7)$ & 4,1 & $1,7-9,7$ \\
\hline 50-59 Jahre & $85(81,7)$ & $19(18,3)$ & 5,3 & $2,1-13,5$ \\
\hline$>60$ Jahre & $10(43,5)$ & $13(56,5)$ & 50,6 & $15,8-162$ \\
\hline \multicolumn{5}{|l|}{ Migration } \\
\hline Geboren in Deutschland & $603(91,8)$ & $54(8,2)$ & & \\
\hline Immigrant & $136(82,9)$ & $28(17,1)$ & 2,4 & $1,4-4,2$ \\
\hline \multicolumn{5}{|l|}{ Tätigkeit } \\
\hline nicht medizinisches Personal & $302(90,1)$ & $33(9,9)$ & 1 & - \\
\hline Krankenschwestern & $334(89,1)$ & $41(10,9)$ & 1,4 & $0,8-2,4$ \\
\hline Ärzte & $103(92,8)$ & $8(7,2)$ & 0,9 & $0,3-2,3$ \\
\hline \multicolumn{5}{|l|}{ TB in Anamnese } \\
\hline keine TB & $732(90,4)$ & $78(9,6)$ & 1 & - \\
\hline ТВ & $7(63,6)$ & $4(36,4)$ & 3,1 & $1,5-6,7$ \\
\hline \multicolumn{5}{|l|}{ THT } \\
\hline kein THT & $192(92,8)$ & $15(7,2)$ & 1 & - \\
\hline negativ in Anamnese & $334(93,8)$ & $22(6,2)$ & 1,0 & $0,5-2,2$ \\
\hline positiv in Anamnese & $138(82,6)$ & $29(17,4)$ & 3,1 & $1,5-6,7$ \\
\hline neuer $\mathrm{THT} \geq 10 \mathrm{~mm}$ & $75(82,4)$ & $15(17,6)$ & 2,8 & $1,2-6,7$ \\
\hline
\end{tabular}

Bei Beschäftigten in der Geriatrie ist der IGRA etwa doppelt so häufig positiv als bei anderen Beschäftigten im Gesundheitsdienst (Odds Ratio 2,4; 95\%KI 1,1-5,2). Da in der Berechnung das Alter und andere Risikofaktoren für eine LTBI kontrolliert wurden, spricht diese Beobachtung für ein erhöhtes Infektionsrisiko der Beschäftigten in der Geriatrie [59].

\section{Beweiserleichterung im Berufskrankheitenverfahren}

Tuberkuloseinfektionen können nach der Nr. 3101 der Anlage zur Berufskrankheitenverordnung als Berufskrankheit anerkannt und entschädigt werden. Dies betrifft Versicherte, die in Arbeitsbereichen des Gesundheitsdienstes, der Wohlfahrtspflege und in Laboratorien tätig sind, in denen eine erhöhte Infektionsgefahr besteht. Bei Versicherten, die nicht in diesen Gefährdungsbereichen arbeiten, sind die Voraussetzungen für eine Anerkennung als Arbeitsunfall im Einzelfall zu prüfen [60].

Trotz der in der BK-Nr. 3101 geforderten Zugehörigkeit zu einer grundsätzlich besonders gefährdeten Personengruppe reicht es nach gesicherter Rechtsprechung für die Anerkennung einer Berufskrankheit nach der BK-Nr. 3101 nicht aus, dass der Versicherte einer der genannten Personengruppen angehört. Vielmehr muss auch unter dem Aspekt einer Berufskrankheit nach BKNr.3101 im Einzelfall festgestellt werden, ob die Infektionskrankheit durch die berufliche Tätigkeit verursacht wurde [61]. Der Beweismaßstab der Wahrscheinlichkeit für den haftungsbegründenden und den haftungsausfüllenden Ursachenzusammenhang gilt sowohl für Arbeitsunfälle als auch für Berufskrankheiten. Es ist allerdings typisch für eine Reihe von Berufskrankheiten, dass ein etwaiger Ursachenzusammenhang zwischen einer gesundheitsgefährdenden beruflichen Exposition und einer später tatsächlich eingetretenen Erkrankung allein anhand einer Beobachtung des Einzelfalles nicht nachvollziehbar ist. Eine Beweisführung unter Berücksichtigung des generellen wissenschaftlichen Erfahrungswissens über Krankheitsursachen und spezifische Risikoerhöhungen bei typischen Tätigkeiten spielt daher im Berufskrankheitenrecht eine besondere Rolle. Das zum 01.01.1997 in Kraft getretene SGB VII greift diesen Gesichtspunkt durch folgende besondere Beweisführungsvorschrift auf:

§9 Abs. 3 SGB VII

Erkranken Versicherte, die infolge der besonderen Bedingungen ihrer versicherten Tätigkeit in erhöhtem Maße der Gefahr der Erkrankung an einer in der Rechtsverordnung nach Abs. 1 genannten Berufskrankheit ausgesetzt waren, an einer solchen Krankheit und können Anhaltspunkte für eine Verursachung außerhalb der versicherten Tätigkeit nicht festgestellt werden, wird vermutet, dass diese infolge der versicherten Tätigkeit verursacht worden ist.

In der Praxis der Berufsgenossenschaft für Gesundheitsdienst und Wohlfahrtspflege (BGW) wurde bislang eine solche über das gewöhnliche Maß deutlich hinausgehende Gefährdung bezüglich einer Tbc-Infektion vor allem bei Beschäftigten in Lungenfachkliniken oder beim Personal auf Infektionsstationen, sofern nach gesicherter Erfahrung dort häufig Tbc-Patienten behandelt werden, angenommen. Entsprechendes galt für lungenfachärztliche Praxen nach Maßgabe näherer Ermittlungen. Eine weitere besonders gefährdete Fallgruppe waren Beschäftigte aus medizinischen Laboratorien, die mit der Untersuchung auf Mykobakterien befasst sind. Im Rahmen der Qualitätssicherung bei der Begutachtung von Berufskrankheiten wurden auf einer Expertentagung [62] die Kriterien für eine Beweiserleichterung unter Berücksichtigung der obengenannten Literaturauswertung überarbeitet. Seither wird bei folgenden Tätigkeiten bzw. in folgenden Bereichen eine über das gewöhnliche Maß deutlich hinausgehende Gefährdung angenommen: 


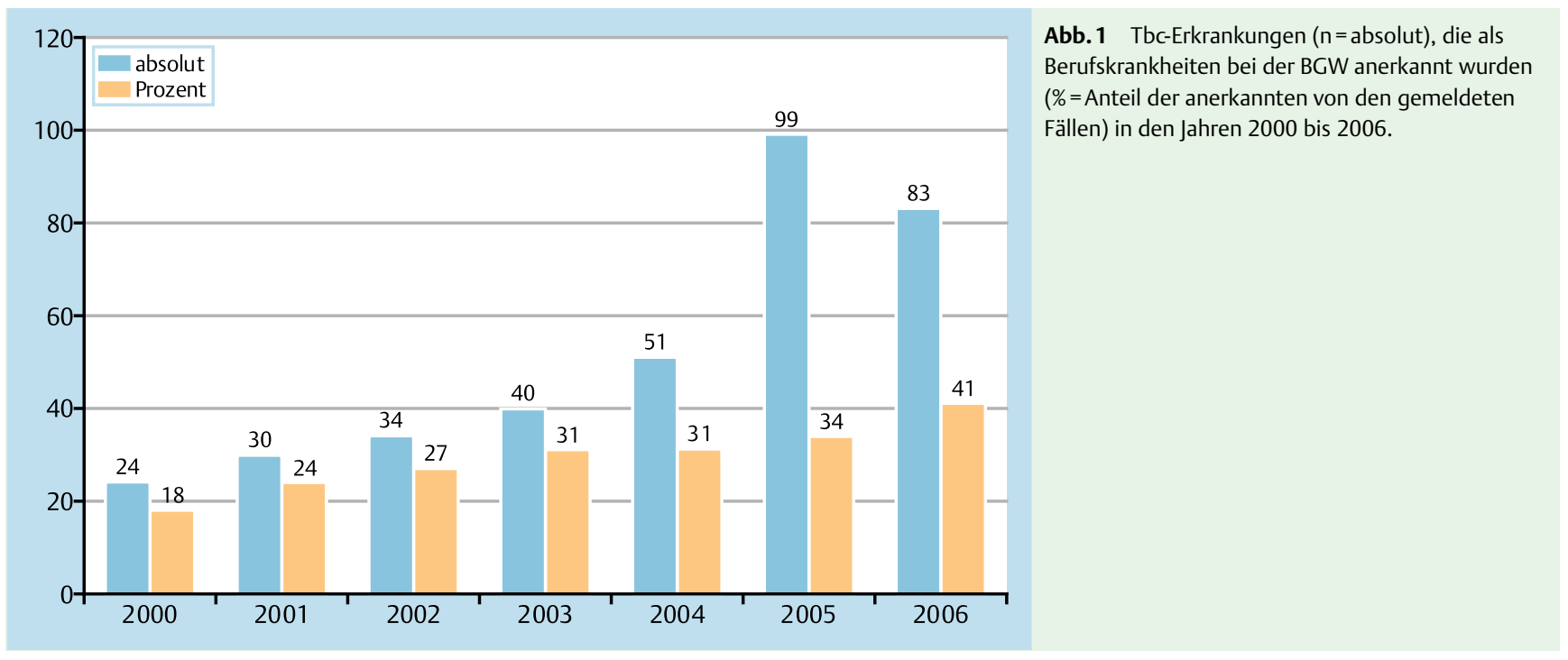

- auf Tbc-Stationen, in Lungenfachkliniken und -praxen (auch Physiotherapeuten mit Atemtherapie, wenn sie in diesen Einrichtungen arbeiten)

- in Laboratorien, die Sputumproben untersuchen (die Gefahr ist dann besonders groß, wenn man nicht an Tbc denkt)

- auf Infektionsstationen

- bei der Bronchoskopie, Kehlkopfspiegelung und Notfallintubation

- in Sektionsräumen der Pathologie und Rechtsmedizin

- in Notaufnahmen, Rettungsdiensten

- bei der Betreuung von Hochrisikogruppen (HIV, Drogen- und Alkoholabhängige, Gefängnisinsassen, Obdachlose, Immigranten aus Ländern mit hoher Tbc-Inzidenz)

- bei Auslandseinsätzen in Gebieten mit bekannt hoher TbcInzidenz

- seit 2007 ist diese Auflistung um Beschäftigte in der Altenpflege und Geriatrie erweitert worden [59].

Mit der Einführung dieser Beweiserleichterungskriterien wird dem Kenntnisstand der aktuellen beruflichen Tbc-Epidemiologie Rechnung getragen. Durch den Verzicht auf den Nachweis einer Indexperson und die Gewährung von Beweiserleichterung wird außerdem das Berufskrankheitenverfahren in vielen Fällen vereinfacht, da die Suche nach einer Indexperson im Nachhinein oftmals sehr zeitaufwendig ist. Durch die Anwendung dieser Beweiserleichterungskriterien hat sich die Anerkennungsrate bei den gemeldeten Tuberkulosen von 18\% im Jahr 2000 auf $41 \%$ im Jahr 2006 bei der BGW mehr als verdoppelt ( $\bullet$ Abb. 1).

Der bisherige Beitrag der betriebsärztlichen Vorsorgeuntersuchungen nach Biostoffverordnung zur frühzeitigen Fallfindung und damit zur Unterbrechung von Infektionswegen ist unbekannt, da weder Konversionen noch aktive, behandlungsbedürftige Tbc-Erkrankungen, die über diese Untersuchungen entdeckt wurden, dokumentiert werden. Entsprechend der Biostoffverordnung werden Beschäftigte auf Tbc-Stationen und in Labors, die regelmäßig Kontakt zu Tbc-Patienten haben oder Proben auf Tbc untersuchen, regelmäßig untersucht. Allen anderen Beschäftigten muss nach einem Kontakt zu einem Tbc-Patienten vom Arbeitsgeber eine Vorsorgeuntersuchung angeboten werden (Biostoffverordnung $\S 15$ Abs.2). Diese Angebotsuntersuchungen durch den Betriebsarzt erfolgen am besten in Absprache mit dem zuständigen Gesundheitsamt entsprechend den
Empfehlungen zur Durchführung von Umgebungsuntersuchungen des DZK [64]. Diese Angebotsuntersuchungen können, sofern sie wahrgenommen werden, die Umgebungsuntersuchungen nach dem Infektionsschutzgesetz ersetzen. Das IfSG hat zum Ziel, die Eigenverantwortung der Träger von Gemeinschaftseinrichtungen, Lebensmittelbetrieben, Gesundheitseinrichtungen sowie des Einzelnen bei der Prävention übertragbarer Erkrankungen zu fördern (IfSG $§ 1$, Satz 2). Die Gesundheitsämter müssen daher die Untersuchungen nicht selber ausführen, wenn andere Stellen „aufgrund anderweitiger gesetzlicher Vorschriften (...) zur Kostentragung verpflichtet sind“ ( $\S 69$, Satz1). Mit dem Betriebsarzt und der aufgrund der Biostoffverordnung anzubietenden Untersuchungen ergibt sich genau so eine Stelle, die die Untersuchungen bereits kompetent und sachgemäß ausführt. Der umgekehrte Weg ist nicht möglich: Eine Umgebungsuntersuchung nach IfSG kann das Angebot einer Untersuchung nach Biostoffverordnung durch den Arbeitgeber nicht ersetzen, da das Arbeitsschutzgesetz als das der Biostoffverordnung übergeordnete Gesetz eine derartige Ablösung der gesetzlichen Verpflichtung des Arbeitgebers nicht vorsieht. Im Berufskrankheitenverfahren wird für weitere Tätigkeiten (Aufnahmestation, Rettungsdienst, Endoskopie, Altenpflege usw.) eine gegenüber der Normalbevölkerung erhöhte Infektionsgefährdung angenommen. Das Anerkennungsverfahren wird dadurch erleichtert. Diese Personen werden dennoch zurzeit nicht regelmäßig entsprechend der Biostoffverordnung untersucht. Um die Anzahl der Anlass bezogenen (Angebots)-Untersuchungen oder Umgebungsuntersuchungen zu reduzieren, kann es sinnvoll sein, je nach örtlichen Gegebenheiten auch in diesen gefährdeten Bereichen regelmäßige Vorsorgeuntersuchungen durchzuführen.

Die Frage, ob die regelmäßigen Vorsorgeuntersuchungen durch die Betriebsärzte nicht besser auf alle Bereiche ausgedehnt werden sollten, bei denen von einer erhöhten Gefährdung insbesondere durch (noch) nicht erkannte Tbc-Patienten ausgegangen werden kann, lässt sich im Moment mangels Daten nicht befriedigend beantworten. Die zurzeit weitergeführte Studie zur Evaluation des IGRA bei Beschäftigten im Gesundheitswesen $[54,55,57,59]$ wird voraussichtlich für eine derartige Entscheidung eine bessere Datengrundlage schaffen. 


\section{Literatur}

1 Statistisches Bundesamt. Fachserie 12, Reihe 2 (meldepflichtige Krankheiten). 2000

2 Robert Koch-Institut. Bericht zur Epidemiologie der Tuberkulose in Deutschland für 2005. Berlin, 2007

3 Schönberger A, Mehrtens G, Valentin H. Arbeitsunfall und Berufskrankheit - rechtliche und medizinische Grundlagen für Gutachter, Sozialverwaltung, Berater und Gerichte, 6. neubearbeitete Auflage. Berlin, Bielefeld, München: Erich Schmidt Verlag, 1998: 748

4 Jentgens $H$, Wandelt-Freerksen $E$. Überlegungen zur Begutachtung der Tuberkulose und deren Folgen im Versicherungswesen. Med Sach 1993; 89: $117-121$

5 Loddenkemper R, Sagebiel D, Brendel A. Strategies against multidrugresistant tuberculosis. Eur Resp J 2002; 20 (Suppl. 36): 66s - 77s

6 Niemann S, Rüsch-Gerdes S, Richter E. IS6610 Fingerprinting of drugresistant Mycobacterium tuberculosis strains isolated in Germany during 1995. J Clin Microbiol 1997; 35: 3015 - 3020

7 Menzies D, Fanning A, Yuan L et al. Tuberculosis among health care workers. N Engl J Med 1995; 332: 92 - 98

$8 C D C$ - Centers for Disease Control. The use of preventive therapy for tuberculosis infection in the United States - Recommendations of the Advisory Committee for Elimination of Tuberculosis. MMWR 1990; 39 (RR-8): $9-12$

9 Burgos MV, Pym AS. Molecular epidemiology of tuberculosis. Eur Respir J Suppl 2002; 36: 54s-65s

10 Soolingen D van, Borgdorff MW, de Haas PE et al. Molecular epidemiology of tuberculosis in the Netherlands: a nationwide study from 1993 through 1997. J Infect Dis 1999; 180: 726 - 736

11 Borgdorff MW, Nagelkerke NJ, de Haas PE et al. Transmission of Mycobacterium tuberculosis depending on the age and sex of source cases. Am J Epidemiol 2001; 154: 934 - 943

12 Diel R, Schneider S, Meywald-Walter K et al. Epidemiology of tuberculosis in Hamburg, Germany: long-term population-based analysis applying classical and molecular epidemiological techniques. J Clin Microbiol 2002; 40: 532-539

13 Cattamanchi A, Hopewell PC, Gonzalez LC et al. A 13-year molecular epidemiolgical analysis of tuberculosis in San Francisco. Int J Tuberc Lung Dis 2006; 10: 297-304

14 Keskiner R, Ergönül O, Demiraglu Z et al. 1. Risk of tuberculous infection among healthcare workers in a tertiary-care hospital in Ankara, Turkey. Infect Control Hosp Epidemiol 2004; 25: 1067-1071

15 Hosoglu S, Tanrikulu AC, Dagli C et al. Tuberculosis among health care workers in a short working period. Am J Infect Control 2005; 33: 23 26

16 Mitchell CS, Gershon RR, Lears MK et al. Risk of tuberculosis in correctional healthcare workers. J Occup Environ Med 2005; 47: 580 586

17 Laniado-Laborin R, Cabrales-Vargas N. Tuberculosis in healthcare workers at a general hospital in Mexico. Infect Control Hosp Epidemiol 2006; 27: 449-452

18 Baussano I, Bugiani M, Carosso A et al. Risk of tuberculin conversation among healthcare workers and the adoption of preventive measures. Occup Environ Med 2007; 64: 161 - 166

19 Seidler A, Nienhaus A. Epidemiologie der berufsbedingten Tbc - Ein Literaturreview zu konventionellen Studien. In: Nienhaus A, Brandenburg S, Teschler H (eds). Tuberkulose als Berufskrankheit - Ein Leitfaden zur Begutachtung. Landsberg: Ecomed, 2003, Kap.12

20 Seidler A, Nienhaus A, Diel R. Review of epidemiological studies on the occupational risk of tuberculosis in low-incidence areas. Respiration 2005; 72: $431-446$

21 Hill A, Burge A, Skinner C. Tuberculosis in National Health Service hospital staff in the west Midlands region of England 1992 - 1995. Thorax 1997; 52: $994-997$

22 Menzies D, Fanning A, Yuan L et al. Hospital ventilation and risk for tuberculous infection in Canadian health care workers. Canadian Collaborative Group in Nosocomial Transmission of TB. Ann Intern Med 2000; 133: 779-789

23 Meredith S, Watson JM, Citron KM et al. Darbyshire: Are healthcare workers in England and Wales at increased risk of tuberculosis? BMJ 1996; 313: $522-525$

24 Schwartzman $K$, Loo V, Pasztor J et al. Tuberculosis infection among health care workers in Montreal. Am J Respir Crit Care 1996; 154: $1006-1012$
25 Stuart RL, Bennett NJ, Forbes AB et al. Assessing the risk of tuberculosis infection among healthcare workers: the Melbourne Mantoux Study. The Melbourne Mantoux Study Group. Med J Aust 2001; 174: 569573

26 Panlilio AL, Burwen DR, Curtis AB et al. Tuberculin skin testing surveillance of health care personnel. Clin Infect Dis 2002; 35: 219-227

27 McKenna MT, Hutton M, Cauthen $G$ et al. The association between occupation and tuberculosis. A population-based survey. Am J Respir Crit Care Med 1996; 154: 587-593

28 Berman JM, Levin L, Orr TS et al. Tuberculosis risk for hospital employees: analysis of a five year tuberculin skin testing program. Am J Public Health 1981; 71: $1217-1222$

29 Christie $C D$, Constantinou P, Marx ML et al. Low risk for tuberculosis in a regional pediatric hospital: nine-year study of community rates and the mandatory employee tuberculin skintest program. Infect Control Hosp Epidemiol 1998; 19: 168 - 174

30 Menzies D, Fanning A, Yuan L et al. Canadian Collaborative Group in Nosocomial Transmission of Tuberculosis. Factors associated with tuberculin conversion in Canadian microbiology and pathology workers. Am J Respir Crit Care Med. 2003; 167: 599-602

31 Kralj N, Hofmann F, Michaelis M. Zur Methodik der Tuberkulosefrüherkennung bei arbeitsmedizinischen Vorsorgeuntersuchungen im Gesundheitsdienst. Arbeitsmed Sozialmed Umweltmed 1997; 32: 5054

32 Plitt SS, Soskolne CL, Fanning A et al. Prevalence and determinants of tuberculin reactivity among physicians in Edmonton, Canada: 1996 - 1997. Int J Epidemiol 2001; 30: 1022 - 1028

33 Ussery XT, Bierman JA, Valway SE et al. Transmission of multidrug-resistant Mycobacterium tuberculosis among persons exposed in a medical examiner's office, New York. Infect Control Hosp Epidemiol 1995; $16: 160-165$

34 Warren DK, Foley KM, Polish LB et al. Tuberculin skin testing of physicians at a midwestern teaching hospital: a 6-year prospective study. Clin Infect Dis 2001; 32: 1331-1337

35 Boudreau AY, Baron SL, Steenland NK et al. Occupational risk of Mycobacterium tuberculosis infection in hospital workers. Am J Ind Med 1997; 32: $528-534$

36 Zarzuela Ramirez M, Dona CJA, Milla EP et al. Influencia del criterio de análisis sobre la incidencia y los factores de riesgo de conversión tuberculínica en personal sanitario. Med. Clin (Barc) 2000; 114: 493 495

37 Liss GM, Khan R, Koven $E$ et al. Tuberculosis infection among staff at a Canadian community hospital. Infect Control Hosp Epidemiol 1996; 17: $29-35$

38 Niemann S. Molekularbiologische Charakterisierung von Mycobacterium tuberculosis-Isolaten. In: Nienhaus A, Brandenburg S, Teschler $\mathrm{H}$ (eds). Tuberkulose als Berufskrankheit - Ein Leitfaden zur Begutachtung. Landsberg: Ecomed, 2003, Kap. 9

39 Sepkowitz KA, Friedman CR, Hafner A et al. Tuberculosis among urban health care workers: a study using restriction fragment length polymorphism typing. Clin Infect Dis 1995; 21: $1098-1101$

40 Deutekom H van, Gerritsen JJJ, Soolingen D van et al. A molecular epidemiological approach to studying the transmission of tuberculosis in Amsterdam. Clin Infect Dis 1997; 25: $1071-1077$

41 Diel R, Seidler A, Nienhaus A et al. Occupational risk of tuberculosis transmission in a low incidence area. Respir Res 2005; 6: 35-45

42 deVries G, Sebek MMGG, Lambregts-van Weezenbeek CSB. Healthcare workers with tuberculosis infected during work. Eur Respir J 2006; 28: $1216-1221$

43 Ong A, Rudoy I, Gonzalez LC et al. Tuberculosis in Healthcare Workers: A molecular epidemiologic study in San Francisco. Infect Control Hosp Epidemiol 2006; 27: $453-458$

44 Kenyon TA, Ridzon R, Luskin-Hawk $R$ et al. Nosocomial outbreak of multidrug-resistant tuberculosis. Ann Intern Med 1997; 127: 32 - 36

45 Haas D, Milton WS, Kreiswirth BN et al. Nosocomial transmission of a drug-sensitive $\mathrm{W}$-variant Mycobacterium tuberculosis strain among patients with acquired immunodeficiency syndrome in Tennessee. Infect Control Hosp Epidemiol 1998; 19: 635-639

46 Moro ML, Gori A, Errante Infuso A et al. An outbreak of multidrug-resistant tuberculosis involving HIV-infected patients of two hospitals in Milan, Italy. Italian Multidrug-Resistant Tuberculosis Outbreak Study Group. AIDS 1998; 12: 1095-1102

47 Peerbooms PG, Doornum GJ van, Deutekom $H$ van et al. Laboratoryacquired tuberculosis. Lancet 1995; 345: 1311 - 1132 
48 Templeton GL, Illing LA, Young L et al. The risk for transmission of Mycobacterium tuberculosis at the bedside and during autopsy. Ann Intern Med 1995; 122: 922 - 925

49 Johnson KR, Braden CR, Cairns KL et al. Transmission of Mycobacterium tuberculosis from medical waste. JAMA 2000; 284: 1683-1688

50 Behr MA, Warren SA, Salamon H et al. Transmission of Mycobacterium tuberculosis from patients smear-negative for acid-fast bacilli. Lancet 1999; 353: 444-449

51 Bennett DE, Onorato IM, Ellis BA et al. DNA Fingerprinting of Mycobacterium tuberculosis isolates from epidemiologically linked case pairs. Emerg Infect Dis 2002; 8: 1224-1229

52 Hayward AC, Goss S, Drobniewski F et al. The molecular epidemiology of tuberculosis in inner London. Epidemiol Infect 2002; 128: 175-184

53 Pai M, Zwerling A, Menzies D. Systematic review: T-Cell-based asays for the diagnosis of latent tuberculosis infection: An update. Ann Intern Med 2008; 149: 177- 184

54 Nienhaus A, Loddenkemper R, Hauer B et al. Latente Tuberkulose Infektion bei Beschäftigten im Gesundheitswesen - Evaluation des Interferon-gamma Release Assay. Pneumologie 2007; 61: 219-223

55 Nienhaus A, Schablon A, Siano B et al. Evaluation of the Interferongamma Release Assay in Healthcare Workers. Int Arch Occup Environ Health 2008; 81: 295-300

56 Soberg $B$, Andersen $A B$, Larsen $H K$ et al. Detecting a low prevalence of latent tuberculosis among health care workers in Denmark detected by M. tuberculosis specific INF- $\gamma$ whole-blood test. Scandinavian Journal of Infectious Diseases 2007; 39: 554 - 559

57 Schablon A, Bauersfeld C, Beckmann G et al. Prävalenz der latenten Tuberkulose-Infektion bei Beschäftigten im Krankenhaus. In: Deutsches Zentralkomitee zur Bekämpfung der Tuberkulose 31. Informationsbericht. Berlin, 2008

58 Nienhaus A, Schablon A, Diel R. Interferon-Gamma Release Assay for the Diagnosis of Latent TB Infection - Analysis of discordant results, when compared to the Tuberculin Skin Test. PloS ONE 2008; 3: e2665

59 Nienhaus A, Schablon A, Loddenkemper $R$ et al. Erhöhte Prävalenz der latenten Tuberkulose Infektion bei Beschäftigten in der Geriatrie? Pneumologie 2007; 61: 613-616
60 Brandenburg S. Rechtliche Voraussetzungen für die Anerkennung und Entschädigung der Tbc als Berufskrankheit. In: Nienhaus A, Brandenburg S, Teschler H (eds). Tuberkulose als Berufskrankheit - Ein Leitfaden zur Begutachtung. Landsberg: Ecomed Verlagsgesellschaft, 2003, Kap. 5

61 Bundessozialgericht - BSG. BSG Urteil v. 29.1.1974. Zeitschrift für Sozialversicherung. 118; BSG-Urteil v. 27.2.1985; HVBG-Info 1985, 31 (1974). 1985

62 Nienhaus A. Berufliches Infektionsrisiko für Tuberkulose - Zusammenfassung und Ergebnisse des Expertengespräches am 12. Mai 2003. In: Nienhaus A, Brandenburg S, Teschler H (eds). Tuberkulose als Berufskrankheit - Ein Leitfaden zur Begutachtung. Landsberg: Ecomed, 2003, Kap.15

63 Greenaway C, Menzies D, Fanning A et al. The Canadian Collaborative Group in Nosocomial Transmission of Tuberculosis. Delay in diagnosis among hospitalized patients with active tuberculosis - predictors and outcomes. Am J Respir Crit Care 2002; 165: 927 - 933

64 Diel $R$, Forßbohm M, Loytved $G$ et al. Empfehlungen für die Umgebungsuntersuchungen bei Tuberkulose - Deutsches Zentralkommitee zur Bekämpfung der Tuberkulose. Pneumologie 2007; 61: 440 - 455

\section{Bisher erschienene Beiträge aus dieser Serie}

1 Murray J. Die historische Entwicklung der Tuberkulose seit Robert Kochs Entdeckung des Tuberkelbazillus 1882. Pneumologie 2007; 61: $764-771$

2 Ulrichs T. Immunologie der Tuberkulose und Entwicklungsstand neuer Impfstoffe. Pneumologie 2008; 62: 143-148

3 Kalsdorf B, Strassburg A, Greinert $U$ et al. Klinik und Diagnose der Tuberkulose. Pneumologie 2008; 62: 284-294

4 Rüsch-Gerdes S, Hillemann D. Moderne mykobakteriologische Labordiagnostik. Pneumologie 2008; 62: 533-540

5 Hauer B, Brodhun B, Altmann D et al. Epidemiologie der Tuberkulose in der Welt und in Deutschland. Pneumologie 2008; 62: 583-594 